University of Wollongong

Research Online

Faculty of Social Sciences - Papers (Archive) Faculty of Arts, Social Sciences \& Humanities

2013

The effects of inhibitory control training on alcohol consumption, implicit alcohol-related cognitions and brain electrical activity

Claire Bowley

University of Wollongong, clb027@uowmail.edu.au

Cameron Faricy

University of Wollongong, cfaricy@uow.edu.au

Bronwyn Hegarty

University of New South Wales, bah04@uow.edu.au

Stuart J. Johnstone

University of Wollongong, sjohnsto@uow.edu.au

Janette L. Smith

University of New South Wales

See next page for additional authors

Follow this and additional works at: https://ro.uow.edu.au/sspapers

Part of the Education Commons, and the Social and Behavioral Sciences Commons

Research Online is the open access institutional repository for the University of Wollongong. For further information contact the UOW Library: research-pubs@uow.edu.au 


\title{
The effects of inhibitory control training on alcohol consumption, implicit alcohol- related cognitions and brain electrical activity
}

\begin{abstract}
This study aimed to replicate findings that alcohol consumption and positive implicit beer-related cognitions can be reduced using inhibitory control (IC) training, with the addition of an active training control. Frontal EEG asymmetry, an objective psychophysiological index of approach motivation, was used as a dependent measure to examine training outcomes. Participants were randomly assigned to one of two IC training conditions (Beer NoGo or Beer Go) or a Brief Alcohol Intervention (BAI) (i.e. the active training control). The IC training tasks consistently paired a stimulus that required a response with images of water (Beer NoGo) or images of beer (Beer Go). Alcohol consumption and implicit beer-related cognitions were measured at pre-training, post-training and at one week follow-up. Frontal EEG asymmetry was recorded during a passive image viewing task that presented neutral, healthy, and beer stimuli - at pre-training, post-training and follow-up. Participants in the Beer NoGo and BAI conditions consumed less beer in a taste test immediately after training than Beer Go participants, suggesting that IC training may be as effective as the already established BAI. The taste test findings were in line with the frontal EEG asymmetry data, which indicated that approach motivation for beer stimuli was altered in the expected directions. However, the positive correlation between post-training frontal EEG asymmetry data and taste test consumption was not significant. While there were no significant changes in implicit beerrelated cognitions following training, a trending positive relationship between implicit beer-related cognitions at post-training and taste test consumption was reported. Further exploration addressing the limitations of the current study is required in order to clarify the implications of these findings.
\end{abstract}

\section{Keywords}

alcohol, consumption, implicit, related, cognitions, brain, effects, electrical, inhibitory, activity, control, training

\section{Disciplines}

Education | Social and Behavioral Sciences

\section{Publication Details}

Bowley, C., Faricy, C., Hegarty, B., Johnstone, S. J., Smith, J. L., Kelly, P. J. \& Rushby, J. A. (2013). The effects of inhibitory control training on alcohol consumption, implicit alcohol-related cognitions and brain electrical activity. International Journal of Psychophysiology, 89 (3), 342-348.

\section{Authors}

Claire Bowley, Cameron Faricy, Bronwyn Hegarty, Stuart J. Johnstone, Janette L. Smith, Peter J. Kelly, and Jacqueline A. Rushby 
The effects of inhibitory control training on alcohol consumption, implicit alcoholrelated cognitions and brain electrical activity

Claire Bowley a

Email: clb027@uowmail.edu.au

Cameron Faricy a

Email: cfaricy@uow.edu.au

Bronwyn Hegarty b

Email: bdhegarty@googlemail.com

Stuart Johnstone ${ }_{\mathrm{a}}$ *

Email: sjohnsto@uow.edu.au

Janette Smith $_{c}$

Email: janette.smith@unsw.edu.au

Peter Kelly a

Email: pkelly@uow.edu.au

Jacqueline Rushby a

Email: j.rushby@unsw.edu.au

a School of Psychology, University of Wollongong,

b School of Psychology, University of New South Wales

c National Drug and Alcohol Research Centre, University of New South Wales

* Corresponding author

Postal address: School of Psychology, University of Wollongong, Wollongong NSW 2522

Australia

Phone: +6124221 4495

Fax: +61242214163 


\section{Abstract}

This study aimed to replicate findings that alcohol consumption and positive implicit beerrelated cognitions can be reduced using inhibitory control (IC) training, with the addition of an active training control. Frontal EEG asymmetry, an objective psychophysiological index of approach motivation, was used as a dependent measure to examine training outcomes. Participants were randomly assigned to one of two IC training conditions (Beer NoGo or Beer Go) or a Brief Alcohol Intervention (BAI) (i.e. the active training control). The IC training tasks consistently paired a stimulus that required a response with images of water (Beer NoGo) or images of beer (Beer Go). Alcohol consumption and implicit beer-related cognitions were measured at pre-training, post-training and at one week follow-up. Frontal EEG asymmetry was recorded during a passive image viewing task that presented neutral, healthy, and beer stimuli - at pre-training, post-training and follow-up. Participants in the Beer NoGo and BAI conditions consumed less beer in a taste test immediately after training than Beer Go participants, suggesting that IC training may be as effective as the already established BAI. The taste test findings were in line with the frontal EEG asymmetry data, which indicated that approach motivation for beer stimuli was altered in the expected directions. However, the positive correlation between post-training frontal EEG asymmetry data and taste test consumption was not significant. While there were no significant changes in implicit beer-related cognitions following training, a trending positive relationship between implicit beer-related cognitions at post-training and taste test consumption was reported. Further exploration addressing the limitations of the current study is required in order to clarify the implications of these findings.

Keywords: Frontal EEG asymmetry; approach motivation; implicit cognitions; inhibitory control training; Go/NoGo task; alcohol 


\section{Introduction}

The prevalence of excessive alcohol consumption among young adults is increasing worldwide (Kypri et al., 2009). In Australia this is particularly noted within undergraduate university students (Hallett et al., 2012). The excessive use of alcohol by this population is concerning, as it is associated with a range of negative health and social consequences (Alcohol Education and Rehabilitation Foundation, 2011; Cox, Hosier, Crossley, Kendall, \& Roberts, 2006; Schuckit, 2009; Teesson et al., 2010). It is therefore important that interventions are developed that help to reduce harmful drinking behaviours. Brief Alcohol Interventions (BAIs) are one example. The aim of BAIs is to reduce alcohol consumption by altering explicit alcohol-related cognitions and behaviours (Palfai \& Ostafin, 2012). Kypri et al. (2009) showed that single session BAIs can reduce university student consumption by up to $17 \%$ after one month and up to $11 \%$ after six months. However, Australian university students have been reported to excessively consume alcohol despite awareness of the potential negative consequences associated with alcohol use (Kypri, et al., 2009; Reavley, Jorm, McCann, \& Lubman, 2011). While BAIs target explicit alcohol-related cognitions (Palfai \& Ostafin, 2012), it remains well documented that implicit alcohol-related cognitions are correlated with alcohol consumption (Houben \& Wiers, 2007a, 2007b, 2008; Payne, Govorun, \& Arbuckle, 2008). This correlation highlights why individuals might consume alcohol despite awareness of potential negative consequences (Wiers \& Stacy, 2006). In light of this possibility, researchers should consider interventions that target implicit cognitions.

Dual process theories of addiction provide insight into alternative ways by which alcohol consumption and implicit cognitions might be altered. Dual process theories suggest drinking behaviour is guided by two cognitive systems: an associative automatic system and a controlled executive system (Bechara, 2005; Strack \& Deutsch, 2004; Wiers et al., 2007). These theories suggest drinking behaviour is activated by automatic processes (e.g. implicit 
cognitions), unless one is able to engage in controlled executive functions - such as inhibitory control (IC). Dual process theories suggest exploration of approaches designed to improve IC, as improved IC should enable better control over automatic tendencies to approach alcohol (Houben, Nederkoorn, Wiers, \& Jansen, 2011; Houben, Wiers, \& Jansen, 2011). Consistent with this perspective, Houben, Nederkoorn, et al. (2011) demonstrated decreased positive implicit beer-related cognitions and alcohol consumption following participation in a Beer NoGo IC training task that consistently paired images of beer with a stopping cue and images of water with a response cue. The opposite results were observed following participation in a training task where the opposite pairing occurred (Beer Go IC training task). Research has suggested that both Go/NoGo manipulations strengthen IC for stimuli paired with a stopping response (Verbruggen \& Logan, 2008), and that the decreased alcohol consumption following Beer NoGo training is mediated by implicit devaluation of alcohol (Houben, Havermans, Nederkoorn, \& Jansen, 2012). Despite these indications, it remains that behavioural changes following IC training may be attributed to reduced approach motivation (Houben, Nederkoorn, et al., 2011).

Previous literature examining the behavioural effects of IC training has relied predominantly on self-report and reaction time measures (Houben, Nederkoorn, et al., 2011; Jones et al., 2011). The addition of objective psychophysiological measures minimises issues associated with these measures (e.g. social desirability, deliberate faking) (Fiedler, Messner, \& Bluemke, 2006; Hofmann, Gawronski, Gschwendner, Le, \& Schmitt, 2005), allowing for examination of the mechanisms underlying the behavioural effects of IC training. The electroencephalogram (EEG) is useful in this context, particularly within the frontal lobes. Examining frontal EEG asymmetry allows consideration of the outcomes of behavioural interventions without the confounding issues generally associated with self-report techniques (Hofmann, et al., 2005), as frontal EEG asymmetry is an objective index of approach 
motivation (Davidson, 1992). Frontal EEG asymmetry is used to index approach motivation via the alpha frequency band $(7.5-12.5 \mathrm{~Hz})$ which is inversely associated with cortical activation (Coan \& Allen, 2003). Greater activity in the left frontal lobe has been associated with approach behaviours (Davidson, 1992; Davidson, Ekman, Frieson, Saron, \& Senulis, 1990; Sutton \& Davidson, 1997). An exploration of frontal EEG asymmetry in this context is warranted, as it has been suggested that alterations in motivation to approach alcohol may explain changes in behaviour following IC training (Houben, Nederkoorn, et al., 2011).

The aim of the current study was to replicate and extend the work of Houben, Nederkoorn, et al. (2011), using a sample of Australian undergraduate university students. In line with Houben, Nederkoorn, et al.(2011), participants were randomly assigned to Beer Go or Beer NoGo IC training conditions. To extend this work, participants were also assigned to an active training control condition that consisted of a BAI (i.e. The THRIVE Program) (Hallett, Maycock, Kypri, Howat, \& McManus, 2009; Kypri, et al., 2009). The effects of these three training tasks on weekly alcohol consumption, implicit beer-related cognitions and beer consumption in a taste test following training were explored. In a further extension, frontal EEG asymmetry was used as a dependent measure of approach motivation, in an attempt to clarify possible mechanisms for alcohol consumption change. Replication of Houben, Nederkoorn, et al.’s (2011) findings would provide support for the notion that IC training could be included as an simple, cost effective addition to interventions that target excessive alcohol consumption.

It was hypothesised that (a) participants assigned to the Beer NoGo and BAI conditions would consume less beer than those in the Beer Go condition during the taste test following training; (b) participants assigned to the Beer NoGo and BAI conditions would decrease their consumption of alcohol and participants assigned to the Beer Go condition would increase their consumption of alcohol, in the week following training; (c) participants 
assigned to the Beer NoGo condition would have less positive implicit beer-related cognitions at post-training and one week follow-up compared to pre-training, while the opposite was expected after participation in the Beer Go training. The effect of BAIs on implicit cognitions was exploratory, as the BAI has yet to be examined within the context of implicit cognitions. It was further hypothesised that (d) participants assigned to the Beer NoGo and BAI conditions would exhibit decreased relative left frontal EEG hemisphere activity during the passive viewing of beer images, indicating reduced approach motivation towards beer. The opposite directional frontal EEG asymmetry changes were predicted for the Beer Go condition.

\section{Method}

\subsection{Participants}

Participants were 59 students from the University of Wollongong, including 39 undergraduate psychology students (11 females) and 20 undergraduate non-psychology students (3 females). Recruitment strategies were consistent with those used by Houben, Nederkoorn, et al. (2011). Recruitment advertisements stated that participants were needed for research on reaction times and attitudes/preferences for beer, and that participants must regularly consume beer and consider beer their preferred alcoholic beverage. Written consent was obtained in accordance with protocol approved by The University of New South Wales Human Research Ethics Committee. Participants were randomly assigned to one of three experimental conditions (Beer NoGo, $n=20,5$ females; Beer Go, $n=20$, 4 females; BAI, $n$ =19, 5 females), and were free to withdraw from the study at any time.

\subsection{Inclusion/exclusion criteria}

Participants were required to be aged between 18 and 30 years $(\mathrm{M}=20.95$, SD = 2.45). Participants completed the Alcohol Use Disorders Identification Test (AUDIT) (Saunders, Aasland, Babor, De La Fuente, \& Grant, 1993) as a screening measure upon 
arrival. Participants were excluded from the study if their AUDIT score was 20 or higher, as this score indicates the person was drinking at high-risk levels. These participants were provided with information on treatment services for problematic alcohol use $(n=3)$. Participants were also excluded if they failed to attend both experimental sessions $(n=7)$ and if they had contraindications to drinking alcohol $(\mathrm{n}=0)$.

\subsection{Go/NoGo Training Tasks}

The Go/NoGo training tasks were completed by participants in the Beer NoGo and Beer Go conditions. The tasks were programmed in Presentation Version 13.1, taking approximately 4 minutes. Participants were instructed to quickly and accurately press the space bar when a Go cue was displayed (50\% of trials) and to refrain from responding when a NoGo cue was displayed (50\% of trials). Go/NoGo cues were the letters ' $\mathrm{P}$ ' and ' $\mathrm{F}$ ' displayed in one of four corners of the stimulus (Figure 1). Assignment of letters to response type was counterbalanced. In the Beer NoGo condition, beer-related images were consistently paired with a NoGo cue and water-related images with a Go cue. The reverse occurred for the Beer Go condition. The task consisted of 80 trials, presented randomly. During each trial, image and cue were presented together in the centre of a black background for $1500 \mathrm{~ms}$ with a response window of $700 \mathrm{~ms}$. A green circle was displayed for $500 \mathrm{~ms}$ after a correct response (i.e. button-press within 700 ms to a Go stimulus; no response to a NoGo stimulus). A red cross was displayed for $500 \mathrm{~ms}$ after an incorrect response (i.e. missed button-press within 700 ms to a Go stimulus; button-press to a NoGo stimulus). There was a blank screen for 1250 ms between stimuli and feedback. Feedback and next trial were separated by a blank screen for $500 \mathrm{~ms}$.

\section{Insert Figure 1}

\subsection{Brief Alcohol Intervention (BAI)}


An Australian BAI, referred to as the THRIVE program (Hallett, et al., 2009; Kypri, et al., 2009), was completed online by participants assigned to the BAI condition. Taking approximately 5 minutes, THRIVE consisted of (a) demographic questions (e.g. gender, age) and the AUDIT; (b) questions about consumption over the previous 4 weeks; and (c) computer generated feedback based on reported alcohol consumption, including AUDIT score, an explanation of the associated health risk, and information about how to reduce this risk.

\subsection{Taste test}

Participants were presented with $330 \mathrm{~mL}$ of chilled beer and $330 \mathrm{~mL}$ of chilled soft drink in clear unlabelled glasses. Beck's non-alcoholic beer was used, however participants were under the assumption the beer was full strength. A fruit-flavoured soft drink was used as these are not normally used as mixers in alcoholic drinks (i.e. lemon squash). Participants were instructed to consume as little or as much of each drink in any order, and to rate their thirst and each drink on five 7-point Likert scales (i.e., not at all thirsty-thirsty, pleasantunpleasant, flat-gassy, bitter-sweet, tasteless-strong tasting), while the researcher left the room for 5 minutes. On returning, the researcher removed the glasses from the participants' view before later measuring how much beer and soft drink had been consumed.

\subsection{Weekly alcohol consumption}

Alcohol consumption in the week before the first experimental session (pre-training) and the week between experimental sessions (follow-up) was assessed using a Timeline Follow-Back (TLFB) interview (Cervantes, Miller, \& Tonigan, 1994). Participants were asked to recall the number, type and size of drinks consumed over the past 7 days. Later, the experimenter converted consumed drinks to Australian standard drinks (Australian Government, 2010).

\subsection{Implicit cognitions}


Implicit beer-related cognitions were measured using an Implicit Association Task (IAT) (Greenwald, McGhee, \& Schwartz, 1998), programmed in Psychology Experiment Building Language (PEBL). The IAT is a computerized classification task based on the assumption that it should be easier to classify two associated concepts using the same response than two unassociated concepts (Greenwald, et al., 1998). The target categories were 'beer' ( 8 beer related images) and 'water' (8 water related images) (Houben, Nederkoorn, et al., 2011). The attribute categories were 'pleasant' (happy, jolly, energetic, funny, sociable, attractive, cheerful, lucky) and 'unpleasant' (dull, miserable, sick, depressed, unhappy, disgusting, angry, vomit) (Houben, Nederkoorn, et al., 2011). The IAT consisted of 6 response blocks (Table 1). Stimuli were presented in the centre of a white background, and remained visible until a correct response was given. If an error occurred a red " $\mathrm{X}$ " appeared above the stimulus item until a correct response was given. Stimulus labels were presented in the upper corners of the computer screen, consistent with response assignment of categories to keys. Participants completed the IAT at pre-training, post-training, and a week later (follow-up).

\section{Insert table 1}

\subsection{Passive image viewing task}

Participants completed a passive image viewing task at pre-training, post-training, and follow-up. The task was programmed in Presentation Version 13.1, and took approximately 8 minutes. Participants were told they would be presented with a sequence of images, to which they were to view. Each task consisted of 30 randomly presently images from 3 categories: neutral (i.e. landscapes); healthy (i.e. glasses of water/juice); beer (i.e. glasses of beer) (Figure 2). Each image was presented for $8000 \mathrm{~ms}$ in the centre of a black background, separated by a blank screen for $8000 \mathrm{~ms}$.

Insert figure 2 


\subsection{Electrophysiological Recording}

EEG was continuously recorded during the passive image viewing task using dry sensor technology, consisting of microchips, embedded firmware, 2 x $10 \mathrm{~mm}$ active electrodes and one ear-clip reference ground electrode (ThinkGear, Neurosky, San Jose, California) contained within a comfortable headband made of stretchable fabric (MindBand, Neurosky, USA). The EEG was recorded continuously from sites Fp1 and Fp2, at $256 \mathrm{~Hz}$. The raw EEG data was sent to a PC via Bluetooth and saved to disc. Research analysing the validity of ThinkGear dry-sensor technology has shown it to be both reliable and valid when compared to research-grade equipment (Johnstone, Blackman, \& Bruggemann, 2012).

\subsection{Procedure}

During the first experimental session (approximately 90 minutes), participants gave their written consent and completed the AUDIT and a demographic questionnaire. The TLFB was administered. Participants completed the IAT and the passive image viewing task while EEG was recorded. Skin conductance and electrocardiogram (ECG) were also recorded, using Ag/AgCl electrodes filled with Biogel placed on distal volar surfaces of digits II and III of the non-dominant hand and pre-jelled Ag/AgCl electrodes placed on the left midline, sternum and right midline - respectively. These were not considered in the current study. Participants were offered a 5 minute break, before completing their assigned training. Participants completed the IAT and passive image viewing task, followed by the taste test.

During the second experimental session one week later (approximately 60 minutes), the TLFB was administered to determine the participants alcohol use over the preceding week. Participants completed the IAT, passive image viewing task, and an awareness probe. All participants were debriefed, and left with a cash reimbursement of \$10. Psychology students were also awarded course credit. Participants received gender specific information 
comparing their alcohol consumption with the Australian population and NHMRC guidelines, and with information about how to reduce consumption.

\subsection{EEG quantification}

Raw EEG data were imported into Neuroscan software. EEG traces were manually inspected and large blink or muscular artifacts excluded. Blink artifacts were removed using the Neuroscan ocular artifact reduction algorithm. The data were epoched from -4000 to 0.0 ms before the presentation of the stimulus (pre-stimulus epoch) and from 250 to $4250 \mathrm{~ms}$ while the stimulus was viewed (post-stimulus epoch). The first $250 \mathrm{~ms}$ post-stimulus was not analysed to avoid eye movement or ERP type artifacts. A Fast Fourier Transform (FFT) using a Hanning filter was used to derive the average power for Delta (1.5 - 3.5 Hz), Theta (3.5 - 7.5 Hz), Alpha $(7.5-12.5 \mathrm{~Hz})$ and Beta $(12.5-25 \mathrm{~Hz})$. The alpha band was examined due to previous evidence linking alpha power to approach motivation (e.g. Davidson et al., 1990). In order to calculate an asymmetry index, the approach outlined in Allen, Coan and Nazarian (2004) was adopted, whereby positive numbers represent increased left hemisphere activity and negative numbers represent increased right hemisphere activity.

\subsection{Statistical analyses}

Statistical analyses were conducted using IBM SPSS Statistics Version 19. Frontal EEG data was subject to Little’s Missing Completely at Random (MCAR) test (Little, 1988), which confirmed that difference scores were missing completely at random EEG $\left(\chi^{2}(9)=\right.$ $.835, p=.98$ ) and at less than 5\%. Therefore, the Expectation Maximisation (EM) process was applied to replace missing values (Dempster, Laird, \& Rubin, 1977). One-way between group analyses of variances (ANOVAs) were used to explore baseline differences on participant characteristics and dependent measures. A one-way analysis of covariance (ANCOVA) was used to explore beer consumption as a percentage of total liquid consumed in the taste test, with AUDIT as the covariate. Session (pre-training, follow-up) by Condition 
(Beer NoGo, Beer Go, BAI) mixed design ANOVAs were used to explore the effects of Condition on weekly alcohol consumption, with repeated measures on the first factor. A Session (pre-training, post-training, follow-up) by Condition (Beer NoGo, Beer Go, BAI) mixed design ANOVA was used to explore the effects of Condition on implicit beer-related cognitions, with repeated measures on the first factor. A Session (pre-training, post-training) by Condition (Beer NoGo, Beer Go, BAI) by Stimulus (neutral, healthy, beer) mixed design ANOVA was used to explore the effect of Condition on frontal EEG asymmetry, with repeated measures on the first factor. Simple effects were explored using a series of paired sample t-tests. Bivariate Pearson's R correlations between dependent variables recorded at post-training and beer consumption in the taste test were used to explore potential mechanisms for consumption change following training. Alpha was set at .05 for all analyses.

\section{Results}

\subsection{Participant characteristics}

Baseline characteristics are shown in Table 2. Given the significant age difference between conditions $(p=.01)$ and the trending AUDIT score difference between conditions ( $p$ $=.06)$, simple effects were explored. Participants assigned to the Beer NoGo $(p=.01)$ and Beer Go $(p=.04)$ conditions were younger than participants assigned to the BAI condition. Participants assigned to the Beer NoGo $(p=.04)$ and Beer Go $(p=.04)$ conditions had lower AUDIT scores than participants assigned to the BAI condition. Given these differences, the primary analyses were repeated with age and AUDIT entered as covariates. The inclusion of age did not alter any of the primary results, while the inclusion of AUDIT altered the taste test results. AUDIT was included as a covariate for the taste test analysis. No participants guessed the aim of the study or the Go/NoGo manipulation, as assessed via an awareness probing questionnaire administered before debriefing. 


\section{Insert table 2}

\subsection{Taste test}

Descriptive statistics for the taste test are shown in Table 3. The main effect of Condition for beer consumption as a percentage of total liquid consumed was significant with AUDIT entered as a covariate $\left(F(2,58)=3.22, p=.05, \eta_{p}{ }^{2}=.11\right)$, with pairwise comparisons subsequently performed. As hypothesised participants assigned to the Beer NoGo $(p=.04)$ and BAI $(p=.03)$ conditions consumed significantly less beer than participants assigned to the Beer Go condition. There was no consumption difference between the Beer NoGo and BAI conditions $(p=.83)$.

\section{Insert table 3}

\subsection{Weekly alcohol consumption}

There were no significant effects of Session or Condition on weekly alcohol consumption, as indicated via the Session by Condition interaction $(F(2,56)=.15, p=.86$, $\left.\eta_{p}{ }^{2}=.01\right)$ and main effects of Session $\left(F(1,56)=.62, p=.44, \eta_{p}{ }^{2}=.01\right)$ and Condition $(F(2$, 56) $\left.=1.32, p=.28, \eta_{p}{ }^{2}=.05\right)$.

\subsection{Implicit cognitions}

IAT effects were calculated with the D600 algorithm (Greenwald, et al., 1998), with higher scores indicating more positive cognitions. There were no significant effects of Session or Condition on implicit beer-related cognitions, as indicated via the Session by Condition interaction $\left(F(4,110)=.31, p=.87, \eta_{p}{ }^{2}=.01\right)$ and main effects of Session $(F(2$, $\left.110)=1.72, p=.18, \eta_{p}{ }^{2}=.03\right)$ and Condition $\left(F(2,55)=1.40, p=.26, \eta_{p}{ }^{2}=.05\right)$.

\subsection{Frontal EEG Asymmetry}

As consumption changes were only observed at post-training in the taste test, the EEG analysis focused on frontal EEG asymmetry difference scores obtained at pre-training and post-training. The Session by Condition interaction approached significance for beer stimuli 
presented during the passive image viewing task $\left(F(2,43)=2.56, p=.09, \eta_{p}{ }^{2}=.11\right)$ (Figure 3). Simple effects revealed that left frontal activity for beer stimuli was increased at posttraining compared to pre-training for the Beer Go condition, which trended towards significance $(p=.06)$. While left frontal activity for beer stimuli was decreased at posttraining compared to pre-training for the Beer NoGo $(p=.50)$ and BAI $(p=.22)$ conditions, these decreases were not significant. There were no significant Session or Condition main effects or interactions for healthy or neutral stimuli.

\section{Insert figure 3}

\subsection{Correlations}

Correlations between dependent measures recorded at post-training and beer consumed in the taste test $(\mathrm{mL})$ were conducted to explore the potential mechanisms for consumption change following training. A positive relationship between frontal EEG asymmetry for beer stimuli at post-training and beer consumed in the taste test was found, however this was not significant $(r=.17, p=.13$ ). A positive relationship between implicit beer-related cognitions at post-training and beer consumed in the taste test was also found, which trended towards significance $(r=.19, p=.08)$ (Figure 4).

\section{Insert figure 4}

\section{Discussion}

The primary aim of the current study was to extend and replicate previous literature examining the effect of IC training on alcohol consumption (Houben, Nederkoorn, et al., 2011). Specifically, this study explored whether IC training could reduce alcohol consumption and compared the outcome to the already established BAI (Hallett, et al., 2009; Kypri, et al., 2009). The effect of IC training was examined within the context of implicit beer-related cognitions and frontal EEG asymmetry. Participants who completed the Beer NoGo training task consumed significantly less beer in the taste test than those in the Beer Go 
condition. This finding was in line with dual process theories, which suggest that approaches designed to improve IC should enable better control over automatic tendencies to approach and consume alcohol (Bechara, 2005; Strack \& Deutsch, 2004; Wiers, et al., 2007). However, IC capacity was not assessed pre- or post- training, so it cannot be reliably claimed that the alcohol consumption changes are the result of altered IC. Alternatively, the alcohol consumption changes following Beer NoGo training may be a result of implicit devaluation of alcohol (Houben, et al., 2012) or reduced approach motivation (Houben, Nederkoorn, et al., 2011). While implicit beer-related cognitions did not significantly change following any training tasks, there was a trending positive relationship between post-training implicit beerrelated cognitions and taste test consumption. This suggests that consumption changes following the training tasks may be attributed to implicit beer-related cognitive changes, in line with Houben et al. (2012). Left hemisphere EEG activity in response to beer stimuli decreased immediately after Beer NoGo training, suggesting decreased approach motivation for beer. However, this decrease was not significant nor was the positive relationship between post-training frontal EEG asymmetry in response to beer stimuli and taste test consumption for all training tasks. Further research that explores the mechanisms of change following the training tasks is therefore required.

Participants who completed the BAI training task consumed significantly less beer in the taste test than those in the Beer Go training task. This was expected as BAIs are an accepted method for reducing alcohol consumption (Kypri, et al., 2009). There was no significant difference in the amount of beer consumed between participants in the Beer NoGo and BAI training conditions. Therefore, the Beer NoGo training task had an effect on immediate beer consumption comparable to an accepted method for reducing alcohol consumption, supporting the notion that IC training could be considered as an addition to interventions that target harmful drinking behaviours. However, this study showed no 
evidence for effects of the Go/NoGo manipulations on consumption in the week following training - suggesting IC training may not be as effective as previous research has indicated (Houben, et al., 2012; Houben, Nederkoorn, et al., 2011). The current study also showed no evidence for an effect of the BAI in the week following the intervention. However, the IC training tasks and BAI took 4 to 5 minutes to complete. It is possible that the training was not long enough to have weekly effects given the length of the experimental session (i.e. approximately 90 minutes). It is also possible that the addition of the passive image viewing task, taking approximately 8 minutes, overrode the effects of training. Despite these limitations, it remains possible that both methods may not be as promising at as previous research has indicated. Future research should explore the effectiveness of both interventions, by considering whether longer or multiple sessions extend the consumption reducing effects. Consideration of the combined effects of IC training and BAIs would also be interesting, as it remains possible that improved executive functioning might enable individuals to better recognise the negative consequences associated with alcohol use (Strack \& Deutsch, 2004), and thus may increase the effectiveness of BAIs. Exploration of the combined effects of IC training and BAIs is further warranted, as all clinically relevant findings following manipulations of implicit processes have combined implicit manipulations with more tradition forms of treatment (Eberl et al., 2013; Schoenmakers et al., 2010; Wiers, Eberl, Rinck, Becker, \& Lindenmeyer, 2011).

Implicit alcohol-related cognitive changes were expected to occur after participation in the Go/NoGo manipulations, following claims drawn from dual process theories of addiction and Houben et al.’s (2012) finding that alcohol consumption changes following Go/NoGo training are mediated by implicit alcohol-related cognitive changes. However, the consumption of the least beer in the taste test by participants who completed the Beer NoGo training was not observed alongside implicit devaluation of beer. The hypothesised increase 
of positive implicit alcohol-related cognitions immediately following participation in the Beer Go training task was also not observed. There were also no implicit alcohol-related cognitive changes following Go/NoGo training observed at follow-up, suggesting there were no longer term effects of IC training on implicit cognitions. These findings suggest that claims drawn from dual process theories and Houben et al.'s (2012) findings may require reconsideration. However, the positive relationship between post-training implicit beer-related cognitions and beer consumption in the taste test suggests that consumption changes following training may indeed be a result of implicit cognitive changes. As this relationship only trended towards significance further exploration is required. Future research should also assess pre-training IC capacity, as the relationship between implicit-alcohol related cognitions may be stronger when IC is reduced (Houben \& Wiers, 2007a, 2007b, 2008, 2009). Additionally, it is possible that pre-training experience effected post-training performance on the IAT, blurring true effects of the training (Nosek, Greenwald, \& Banaji, 2007). Future research should address possible training effects. The current study also found no effect of the BAI on implicit alcohol-related cognitions. However, BAIs aim to reduce alcohol consumption by altering explicit alcohol-related cognitions and behaviours (Palfai \& Ostafin, 2012), which may explain why implicit alcohol-related cognitions were not affected by participation in the BAI.

The current study revealed frontal EEG asymmetry data trending in the expected direction for beer stimuli. The EEG data indicated that left hemisphere activity increased immediately after Beer Go training (which approached significance), suggesting increased approach motivation for beer stimuli. Conversely, left hemisphere activity decreased immediately after Beer NoGo and BAI training. While these decreases were not significant, they were in the expected direction, and suggest decreased approach motivation for beer stimuli. The decrease in left frontal activity following Beer NoGo training was expected, as 
dual process theories suggest tasks designed to improve IC should enable greater control over approach tendencies towards alcohol (Bechara, 2005; Strack \& Deutsch, 2004; Wiers, et al., 2007). The EEG directional changes were also in line with the taste test findings, whereby participants who completed the Beer NoGo and BAI training tasks were found to have consumed significantly less beer in the taste test than those in the Beer Go condition. While the trends from the current study are interesting, additional research exploring the mechanisms of behaviour change following IC training and BAIs is required, as the positive relationship between post-training frontal EEG asymmetry in response to beer stimuli and beer consumption in the taste test was not significant. The current study utilised the IAT and frontal EEG asymmetry to measure implicit beer-related cognitions and approach motivation, as previous literature suggests that the behavioural changes following IC training may be attributed to changes in these variables (Houben, et al., 2012). It has also been noted that behavioural changes following IC training may be attributed to improved IC (Houben, Nederkoorn, et al., 2011). Future researchers should consider implementing additional or alternative dependent measures of implicit cognitions and approach motivation, as well as IC capacity, in order to gain greater insight into the mechanisms by which IC training and BAIs have their behavioural effects. Nonetheless, the EEG directional changes alongside the consumption findings provide further support for the notion that IC training could be considered as an addition to interventions that target harmful drinking behaviours.

In order to accurately replicate Houben, Nederkoorn, et al. (2011), the current study utilised a 50/50 Go/NoGo stimulus presentation ratio. However, Go/NoGo tasks typically require that Go signals are more frequent than NoGo signals (e.g. 70/30). Future research should explore the effects of Go/NoGo manipulations using a typical stimulus presentation ratio. While advertisements stated that individuals who regularly consume beer and consider beer their preferred alcoholic drink were required for participation in the current study, there 
was no cut off for participants who scored low on the AUDIT. As previous research has used participants who scored 8 or higher on the AUDIT (Houben, Nederkoorn, et al., 2011), it is possible that previous findings were not replicated as a result of sample differences.

Therefore, future research should replicate the current study with individuals who score 8 or higher on the AUDIT. Another consideration is motivation for change. The training tasks may have been more effective had participants been identified as being motivated to reduce their alcohol consumption (Hasking, Lyvers, \& Carlopio, 2011). Further, the BAI likely affected motivation to change alcohol consumption, as it aims to reduce alcohol consumption by altering explicit alcohol-related cognitions and behaviours directly (Palfai \& Ostafin, 2012). Future research should assess motivation to reduce alcohol consumption at pre- and post- training when attempting to replicate the current study. Future research could also consider implementing the use of a healthier choice alternative such as water or juice in the taste test, with the IC training tasks utilising stimuli consistent with the alternative beverage. This design could be used to examine if participants can be trained to consume healthier alternatives rather than just conditioning reduced beer consumption.

The current study aimed to replicate and extend previous research examining the effects of IC training on alcohol consumption. In extension, frontal EEG asymmetry was utilised as an objective psychophysiological measure to examine the effects of IC training, with results suggesting approach motivation may have been manipulated post-training. Future research is required before it can be claimed that changes in approach motivation may be responsible for the behavioural effects of training. The positive trending relationship between post-training implicit beer-related cognitions and beer consumption in the taste test suggest that implicit alcohol-related cognitive changes may be responsible for the variation in alcohol consumption. Future research exploring this possibility further is required. The behavioural results suggest that IC training can potentially reduce alcohol consumption 
among Australian undergraduate university students, comparable to the already established BAI. Due to the short duration, simplicity, and possible efficacy of the IC training task, IC training could quickly emerge as a cost-effective adjunct therapy for addressing alcohol misuse among Australian university students. However, longer term effects of IC training on alcohol consumption were not observed. Future research that better establishes the consumption reducing effects of IC training is therefore required. 


\section{References}

Alcohol Education and Rehabilitation Foundation. (2011). Submission to the Federal Government's Tax Forum. Retrieved from http://www.fare.org.au/wpcontent/uploads/2011/07/AER-Foundation-Submission-to-the-Federal-GovernmentsTax-Forum.pdf.

Allen, J. J. B., Coan, J. A., \& Nazarian, M. (2004). Issues and assumptions on the road from raw signals to metrics of frontal EEG asymmetry in emotion. Biol Psychol, 67(1-2), 183-218. doi: 10.1016/j.biopsycho.2004.03.007

Australian Government. (2010). Standard drinks guide. Retrieved from http://www.health.gov.au/internet/alcohol/publishing.nsf/Content/drinksguide-cnt.

Bechara, A. (2005). Decision making, impulse control and loss of willpower to resist drugs: a neurocognitive perspective. Nat Neurosci, 8(11), 1458-1463. doi: 10.1038/nn1584

Cervantes, E. A., Miller, W. R., \& Tonigan, J. S. (1994). Comparison of Timeline FollowBack and Averaging Methods for Quantifying Alcohol Consumption in Treatment Research. Assessment, 1(1), 23-30. doi: 10.1177/1073191194001001004

Coan, J. A., \& Allen, J. J. B. (2003). Frontal EEG asymmetry and the behavioral activation and inhibition systems. Psychophysiology, 40(1), 106-114. doi: 10.1111/14698986.00011

Cox, W. M., Hosier, S. G., Crossley, S., Kendall, B., \& Roberts, K. L. (2006). Motives for drinking, alcohol consumption, and alcohol-related problems among British secondary-school and university students. Addict Behav, 31(12), 2147-2157. doi: 10.1016/j.addbeh.2006.02.023

Davidson, R. J. (1992). Anterior cerebral asymmetry and the nature of emotion. Brain Cognition, 20(1), 125-151. doi: 10.1016/0278-2626(92)90065-T 
Davidson, R. J., Ekman, P. J. A., Frieson, W. V., Saron, C. D., \& Senulis. (1990). Approach/withdrawal and cerebral asymmetry: Emotional expression and brain physiology. International Journal of Personality and Social Psychology, 58(2), 330341. doi: 10.1037/0022-3514.58.2.330

Dempster, A. P., Laird, N. M., \& Rubin, D. B. (1977). Maximum Likelihood from Incomplete Data via the EM Algorithm. J R Stat Soc, 39(1), 1-38.

Eberl, C., Wiers, R. W., Pawelczack, S., Rinck, M., Becker, E. S., \& Lindenmeyer, J. (2013). Approach bias modification in alcohol dependence: Do clinical effects replicate and for whom does it work best? Developmental Cognitive Neuroscience, 4, 38-51. doi: 10.1016/j.dcn.2012.11.002

Fiedler, K., Messner, C., \& Bluemke, M. (2006). Unresolved problems with the “I”, the “A”, and the “T”: A logical and psychometric critique of the Implicit Association Test (IAT). Eur Rev Soc Psychol, 17(1), 74-147. doi: 10.1080/10463280600681248

Greenwald, A. G., McGhee, D. E., \& Schwartz, J. L. K. (1998). Measuring individual differences in implicit cognition: the implicit association test. Journal of Personality and Social Psychology, 74(6), 1464-1480. doi: 10.1037/0022-3514.74.6.1464

Hallett, J., Howat, P. M., Maycock, B. R., McManus, A., Kypri, K., \& Dhaliwal, S. S. (2012). Undergraduate student drinking and related harms at an Australian university: webbased survey of a large random sample. BMC Public Health, 12(1), 37-45. doi: $10.1186 / 1471-2458-12-37$

Hallett, J., Maycock, B., Kypri, K., Howat, P., \& McManus, A. (2009). Development of a Web-based alcohol intervention for university students: Processes and challenges. Drug Alcohol Rev, 28(1), 31-39. doi: 10.1111/j.1465-3362.2008.00008.x 
Hasking, P., Lyvers, M., \& Carlopio, C. (2011). The relationship between coping strategies, alcohol expectancies, drinking motives and drinking behaviour. Addict Behav, 36(5), 479-487. doi: 10.1016/j.addbeh.2011.01.014

Hofmann, W., Gawronski, B., Gschwendner, T., Le, H., \& Schmitt, M. (2005). A MetaAnalysis on the Correlation Between the Implicit Association Test and Explicit SelfReport Measures. Pers Soc Psychol B, 31(10), 1369-1385. doi: $10.1177 / 0146167205275613$

Houben, K., Havermans, R. C., Nederkoorn, C., \& Jansen, A. (2012). Beer à no-go: learning to stop responding to alcohol cues reduces alcohol intake via reduced affective associations rather than increased response inhibition. Addiction, 107(7), 1280-1287. doi: 10.1111/j.1360-0443.2012.03827.x

Houben, K., Nederkoorn, C., Wiers, R. W., \& Jansen, A. (2011). Resisting temptation: Decreasing alcohol-related affect and drinking behavior by training response inhibition. Drug Alcohol Depen, 116(1), 132-136. doi:

10.1016/j.drugalcdep.2010.12.011

Houben, K., \& Wiers, R. W. (2007a). Are drinkers implicitly positive about drinking alcohol? Personalizing the alcohol-IAT to reduce negative extrapersonal contamination. Alcohol Alcoholism, 42(4), 301-307. doi: 10.1093/alcalc/agm015

Houben, K., \& Wiers, R. W. (2007b). Personalizing the alcohol-IAT with individualized stimuli: Relationship with drinking behavior and drinking-related problems. Addict Behav, 32(12), 2852-2864. doi: 10.1016/j.addbeh.2007.04.022

Houben, K., \& Wiers, R. W. (2008). Implicitly positive about alcohol? Implicit positive associations predict drinking behavior. Addict Behav, 33(8), 979-986. doi: 10.1016/j.addbeh.2008.03.002 
Houben, K., \& Wiers, R. W. (2009). Response Inhibition Moderates the Relationship Between Implicit Associations and Drinking Behavior. Alcohol Clin Exp Res, 33(4), 626-633. doi: 10.1111/j.1530-0277.2008.00877.x

Houben, K., Wiers, R. W., \& Jansen, A. (2011). Getting a Grip on Drinking Behavior: Training Working Memory to Reduce Alcohol Abuse. Psychol Sci, 22(7), 968-975. doi: 10.1177/0956797611412392

Johnstone, S. J., Blackman, R., \& Bruggemann, J. M. (2012). EEG From a Single-Channel Dry-Sensor Recording Device. Clinical EEG and neuroscience, 43(2), 112-120. doi: $10.1177 / 1550059411435857$

Jones, A., Guerrieri, R., Fernie, G., Cole, J., Goudie, A., \& Field, M. (2011). The effects of priming restrained versus disinhibited behaviour on alcohol-seeking in social drinkers. Drug Alcohol Depen, 113(1), 55-61. doi: 10.1016/j.drugalcdep.2010.07.006

Kypri, K., Hallett, J., Howat, P., McManus, A., Maycock, B., Bowe, S., \& Horton, N. (2009). Randomized controlled trial of proactive web-based alcohol screening and brief alcohol intervention for university students. Arch Intern Med, 169(16), 1508-1514. doi: 10.1001/archinternmed.2009.249

Little, R. J. A. (1988). A Test of Missing Completely at Random for Multivariate Data with Missing Values. J Am Stat Assoc, 83(404), 1198-1202.

Nosek, B. A., Greenwald, A. G., \& Banaji, M. R. (2007). The implicit association test at age 7: a methodological and conceptual review Automatic processes in social thinking and behaviour (pp. 265-292): Psychology Press.

Palfai, T., \& Ostafin, B. (2012). Implicit cognition as a moderator of brief motivational interventions for alcohol. Addiction Science and Clinical Practice, 7(1), 46. doi: 10.1186/1940-0640-7-S1-A46 
Payne, B. K., Govorun, O., \& Arbuckle, N. L. (2008). Automatic attitudes and alcohol: Does implicit liking predict drinking? Cognition Emotion, 22(2), 238-271. doi: $10.1080 / 02699930701357394$

Reavley, N. J., Jorm, A. F., McCann, T. V., \& Lubman, D. I. (2011). Alcohol consumption in tertiary education students. BMC Public Health, 11(1), 545-564. doi: 10.1186/14712458-11-545

Saunders, J. B., Aasland, O. G., Babor, T. F., De La Fuente, J. R., \& Grant, M. (1993). Development of the Alcohol Use Disorders Identification Test (AUDIT): WHO Collaborative Project on Early Detection of Persons with Harmful Alcohol Consumption-II. Addiction, 88(6), 791-804. doi: 10.1111/j.1360-0443.1993.tb02093.x

Schoenmakers, T. M., de Bruin, M., Lux, I. F. M., Goertz, A. G., Van Kerkhof, D. H. A. T., \& Wiers, R. W. (2010). Clinical effectiveness of attentional bias modification training in abstinent alcoholic patients. Drug and Alcohol Dependence, 109(1-3), 30-36. doi: 10.1016/j.drugalcdep.2009.11.022

Schuckit, M. A. (2009). Alcohol-use disorders. The Lancet, 373(9662), 492-501. doi: 10.1016/S0140-6736(09)60009-X

Strack, F., \& Deutsch, R. (2004). Reflective and Impulsive Determinants of Social Behavior. Pers Soc Psychol Rev, 8(3), 220-247. doi: 10.1207/s15327957pspr0803_1

Sutton, S. K., \& Davidson, R. J. (1997). Prefrontal brain asymmetry: A biological substrate of the behavioural approach and ignition systems. Psychol Sci, 8(3), 204-221.

Teesson, M., Hall, W., Slade, T., Mills, K., Grove, R., Mewton, L., . . Haber, P. (2010). Prevalence and correlates of DSM-IV alcohol abuse and dependence in Australia: findings of the 2007 National Survey of Mental Health and Wellbeing. Addiction, 105(12), 2085-2094. doi: 10.1111/j.1360-0443.2010.03096.x 
Verbruggen, F., \& Logan, G. D. (2008). Automatic and controlled response inhibition: Associative learning in the go/no-go and stop-signal paradigms. J Exp Psychol Gen, 137(4), 649-672. doi: 10.1037/a0013170

Wiers, R. W., Bartholow, B. D., van den Wildenberg, E., Thush, C., Engels, R. C. M. E., Sher, K. J., .. . Stacy, A. W. (2007). Automatic and controlled processes and the development of addictive behaviors in adolescents: A review and a model. Pharmacol Biochem Be, 86(2), 263-283. doi: 10.1016/j.pbb.2006.09.021

Wiers, R. W., Eberl, C., Rinck, M., Becker, E. S., \& Lindenmeyer, J. (2011). Retraining Automatic Action Tendencies Changes Alcoholic Patients' Approach Bias for Alcohol and Improves Treatment Outcome. Psychological Science, 22(4), 490-497. doi: $10.1177 / 0956797611400615$

Wiers, R. W., \& Stacy, A. W. (2006). Implicit Cognition and Addiction. Curr Dir Psychol Sci, 15(6), 292-296. doi: 10.1111/j.1467-8721.2006.00455.x 


\section{Figure and Table Legends}

Figure 1. Examples of (a) beer and (b) water stimuli from the Go/NoGo training tasks.

Table 1. An outline of the IAT response blocks. Categorisations were made using number keys 1 and 2 . In blocks 3 and 6 stimuli belonging to one target category and one attribute category were categorised using the same key (test blocks). Assignment of attribute categories to keys and order of combined sorting conditions were counterbalanced.

Figure 2. Examples of (a) neutral, (b) healthy and (c) beer stimuli from the passive image viewing task.

Table 2. Baseline participant characteristics, for participants assigned to the Beer Nogo, Beer Go and BAI conditions. Values are mean \pm SD.

Table 3. Descriptive statistics for the taste test for participants assigned to the Beer NoGo, Beer Go and BAI conditions.

Figure 3. Mean frontal EEG asymmetry difference scores for beer stimuli presented during the passive image viewing task at pre-training and post-training. Higher scores indicate an increase in left hemisphere activity.

Figure 4. Relationship between implicit beer-related cognitions recorded during the IAT at post-training and $\mathrm{mL}$ of beer consumed in the taste test. Higher implicit beer-related cognition scores indicate more positive cognitions. 


\section{Figures and Tables}

Figure 1

(a)

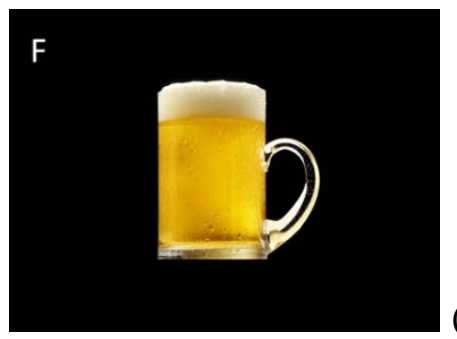

(b)

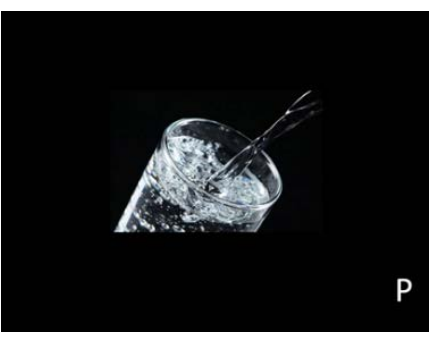

Table 1

\begin{tabular}{|l|l|l|l|}
\hline Block & Categorisations & Trials & Ptimulus \\
\hline 1 & Beer vs. Water & 64 & 4 \\
\hline 2 & Pleasant vs. & 64 & 4 \\
\hline 3 & Unpleasant & & \\
\hline 4 & Beer/Pleasant vs. & 64 & 2 \\
\hline 5 & Water/Unpleasant & & 4 \\
\hline 6 & Water vs. Beer & 64 & 4 \\
\hline & Pleasant vs. & 64 & \\
\hline & Unpleasant & & \\
\hline & Water/Pleasant vs. & 64 & \\
\hline
\end{tabular}


Figure 2

(a)

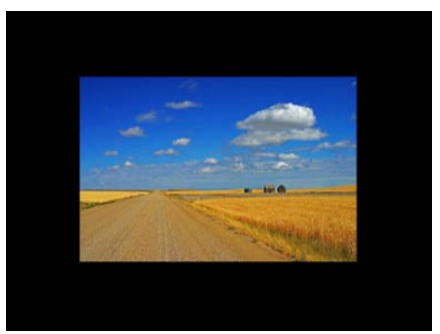

(b)

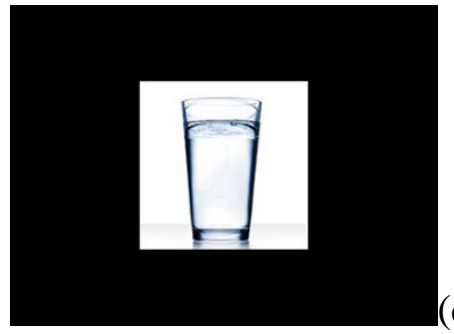

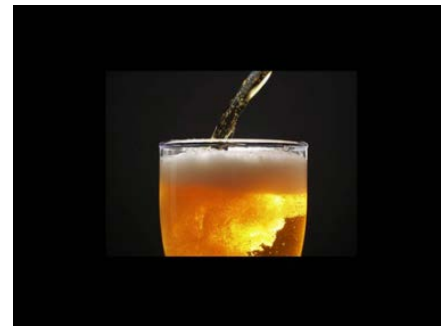

Table 2

\begin{tabular}{|c|c|c|c|c|c|}
\hline & Beer NoGo & Beer Go & BAI & $F(\mathrm{df})$ & $p$ value \\
\hline $\begin{array}{l}\text { Gender ratio } \\
(\mathrm{M}: \mathrm{F})\end{array}$ & $14: 5$ & $16: 4$ & $15: 5$ & & \\
\hline $\begin{array}{l}\text { Student ratio } \\
(\mathrm{P}: \mathrm{NP})\end{array}$ & $12: 7$ & $12: 8$ & $15: 5$ & & \\
\hline Age (years) & $20.05 \pm 1.87$ & $20.32 \pm 1.11$ & $22.21 \pm 3.16$ & $5.09(2,54)$ & .01 \\
\hline AUDIT & $10.37 \pm 4.57$ & $10.40 \pm 3.86$ & $13.00 \pm 3.09$ & $2.89(2,55)$ & .06 \\
\hline TLFB & $\begin{array}{l}12.99 \pm \\
13.83\end{array}$ & $\begin{array}{l}18.65 \pm \\
13.91\end{array}$ & $\begin{array}{l}16.51 \pm \\
11.59\end{array}$ & $.92(2,56)$ & .41 \\
\hline IAT (D600) & $-.18 \pm .38$ & $-.07 \pm .37$ & $-.09 \pm .46$ & $.38(2,55)$ & .69 \\
\hline
\end{tabular}

${ }^{\mathrm{a}} \mathrm{M}$ = male; $\mathrm{F}$ = female; $\mathrm{P}$ = psychology student; $\mathrm{NP}$ = non-psychology student; $\mathrm{AUDIT}$ = total AUDIT score; TLFB = weekly alcohol consumption (standard drinks) estimated with the TLFB; IAT effects were calculated with the D600 algorithm, so higher scores indicate more positive cognitions. 
Table 3

\begin{tabular}{|l|l|l|l|}
\hline & Beer NoGo & Beer Go & BAI \\
\hline Beer consumed (mL) & 184.56 & 234.95 & 168.30 \\
\hline Soft drink consumed & 161.42 & 119.55 & 168.7 \\
(mL) & & & \\
\hline Total liquid & 345.98 & 354.49 & 337.03 \\
consumed (mL) & & & 51.20 \\
\hline Beer as a \% of total & 52.73 & 66.98 & \\
\hline fluid consumed & & & \\
\hline
\end{tabular}

Figure 3

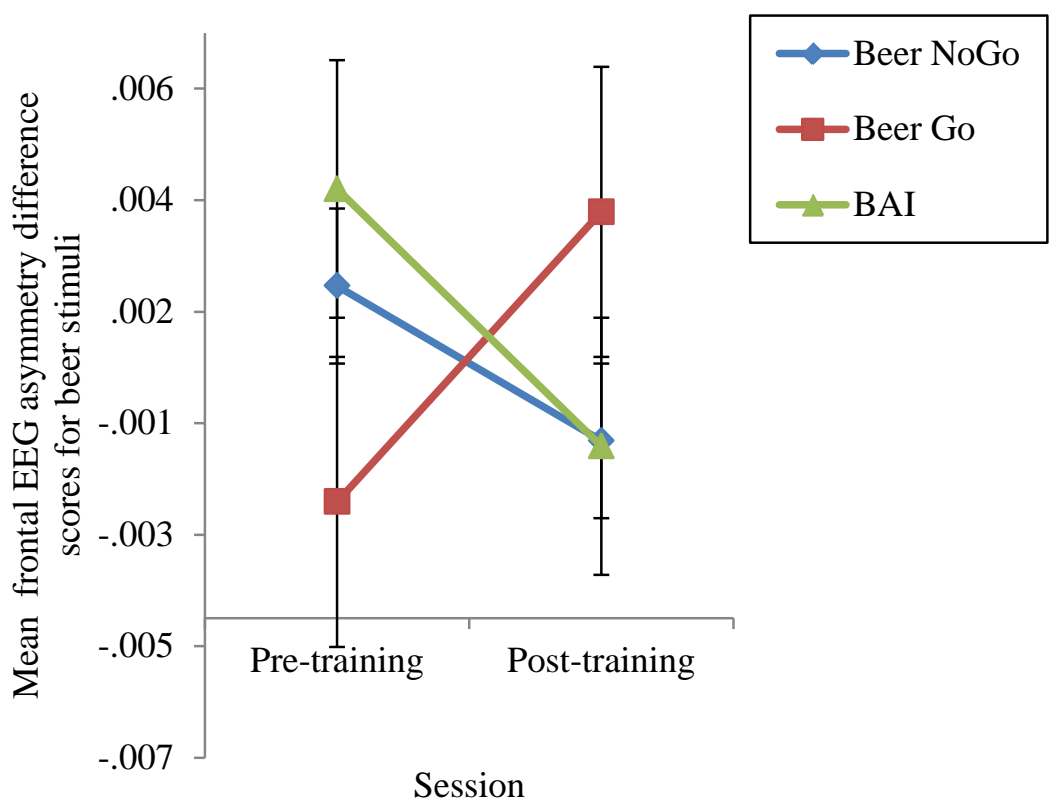


Figure 4

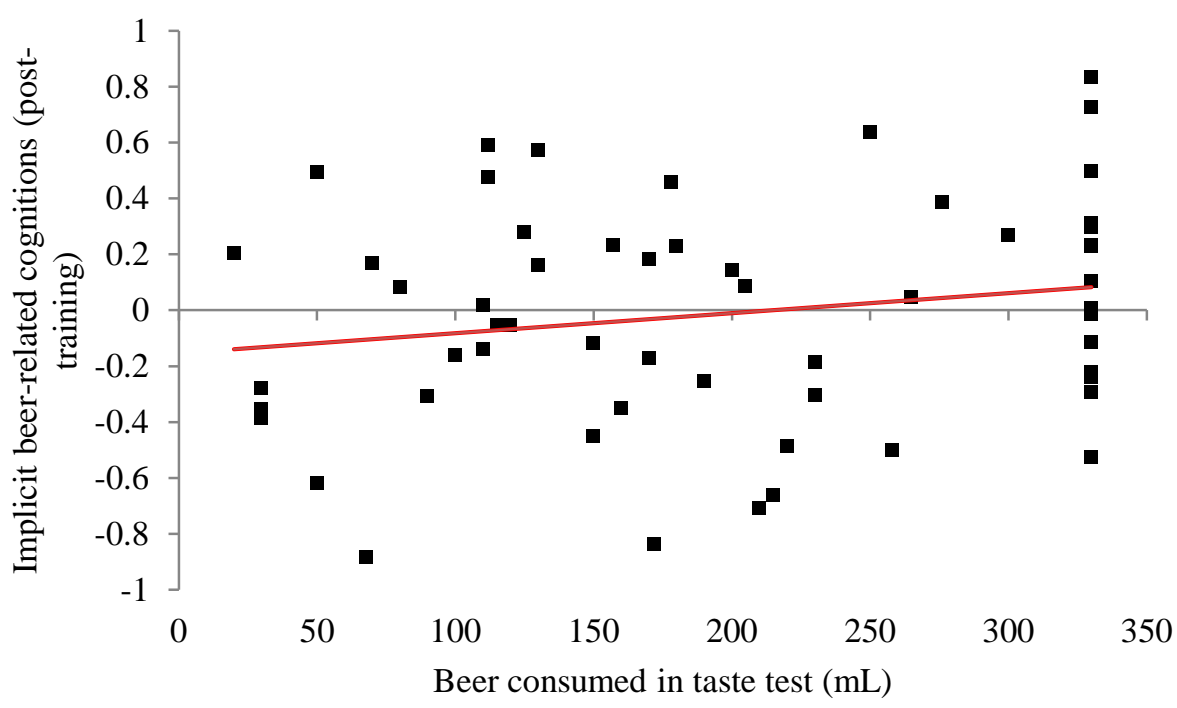

